Cohort study

\section{Pertussis: a significant cause of prolonged acute cough in previously vaccinated school-aged children}

10.1136/ebmed-2014-110072

\section{Michael D Shields}

Centre for Infection \&t Immunity, Queen's University Belfast, Belfast, UK

Correspondence to: Michael D Shields, Centre for Infection \& Immunity, Queen's University Belfast, Health Sciences Building, 97 Lisburn Road, Belfast BT9 7AE, Northern Ireland, UK; m.shields@qub.ac.uk

Commentary on: Wang K, Fry NK, Campbell H, et al. Whooping cough in school age children presenting with persistent cough in UK. BMJ 2014;348:g3668

\section{Context}

Recent evidence suggests that school-aged children with pertussis act as a reservoir for infection in infants who are too young to have been vaccinated and who experience most morbidity. ${ }^{12}$ It has been speculated that the newer acellular vaccines may not protect children for as long as the previous whole-cell vaccine. Owing to the recent epidemic (2011-2013) and some infant deaths from pertussis, the UK now offer pregnant women vaccination, whereas in North America an older child booster was introduced. This study was designed to provide further data on the extent of pertussis in school-aged children, which would inform whether introducing a school age booster vaccination would be worthwhile.

\section{Methods}

This was a prospective 2-year cross-sectional study of 279 children (5-15 years) attending general practice with prolonged acute cough. The case definition was a persistent cough (lasting more than 2 weeks) triggered by an acute upper respiratory tract infection and with other known causes excluded. The authors did not require whooping, vomiting or coughing paroxysms to be part of the case definition. These classical features are known not to add much to the diagnosis of pertussis in adolescents. $^{2}$

Each participant enrolled had a previously validated saliva sample studied for antipertussis toxin IgG. Children were excluded if their last booster vaccination was within the past 12 months to avoid potential false-positive tests. The authors expressed the results descriptively and as risks.

\section{Findings}

Of the 302 children entered into this study 279 yielded suitable saliva samples.

Twenty per cent (95\% CI 16\% to 25\%) or 1 in 5 school-aged children with prolonged acute coughing had evidence of recent pertussis infection. In this study the majority of children enrolled had been previously fully vaccinated (215 of 279) with 18\% having pertussis. The highest risk of pertussis was in older children and especially those who had received their vaccination more than 7 years previously, where the rate was 40\% and the risk was three times higher compared with those receiving recent vaccination.

\section{Commentary}

This study highlights pertussis as a cause of prolonged acute coughing. While the rate of pertussis was high $(20 \%)$ it was lower than their previous study where the rate was $37 \%,{ }^{3}$ and lower than that found by Cornia et $a l^{2}$ who reported a rate of $32 \%$. The study results are generalisable both for primary care and secondary care paediatrics, as the study was large in size and the authors used broad entry criteria; enrolment was not limited to children with the classical whooping cough features (whoop and cough paroxysms followed by vomiting). It is already known that the classical features only add minimally to the diagnosis of pertussis in older children and adolescents. ${ }^{2}$ In addition, the authors used an objective, validated and very suitable diagnostic test.

The authors admit that the general practitioners recruited participants on an opportunistic basis and therefore some were missed. Indeed, based on previous audits they estimated that they captured just over half of all potential participants. Additionally, it is unknown how many school children have prolonged acute cough, perhaps of a milder degree, and never attend their general practitioner. In addition, it is disappointing that the sample did not include enough children with underlying asthma. It can be clinically difficult to sort out whether an asthmatic child whose cough becomes a problem (with overshadowing wheezing symptoms) has; (1) an asthma deterioration, (2) postinfectious cough (like pertussis) or (3) persistent bacterial bronchitis. ${ }^{4}$

The authors' intent was to provide further information to help with the decision as to whether a school age booster vaccine should be introduced in the UK. The high prevalence of pertussis in this group should encourage practitioners in primary and secondary care to think about pertussis. I believe that having a simple diagnostic test on saliva has an added value that the authors do not mention: when I see children with problem cough, it is my experience that if I can objectively confirm a pertussis diagnosis and explain the nature of the illness, I relieve a lot of anxiety and reduce the need for further investigations.

Overall this paper provides very valuable information regarding the aetiology of prolonged acute cough in school-aged children.

\section{Competing interests None.}

Provenance and peer review Commissioned; internally peer reviewed.

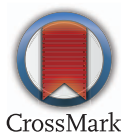

\section{References}

1. Cherry JD. Epidemic pertussis in 2012: the resurgence of a vaccine-preventable disease. N Engl J Med 2012;367:785-90.

2. Cornia PB, Hersh AL, Lipsky BA, et al. Does this coughing adolescent or adult patient have pertussis? JAMA 2010;304:890-6.

3. Harnden A, Grant C, Harrison T, et al. Whooping cough in school aged children with persistent cough: a prospective cohort study in primary care. $B M J$ 2006;33:174-7.

4. Shields MD, Bush A, Everard ML, et al. Recommendations for the assessment and management of cough in children. Thorax 2008;63:1-15. 